\title{
A preclinical study to model taurine pharmokinetics in the undernourished rat
}

\author{
Ana Catalán-Latorre ${ }^{1,2}$, Amparo Nácher ${ }^{1,2}$, Virginia Merino ${ }^{1,2}$, Octavio Díez ${ }^{1,2}$ and \\ Matilde Merino Sanjuán ${ }^{1,2 *}$ \\ ${ }^{1}$ Department of Pharmacy and Pharmaceutical Technology and Parasitology, University of Valencia, 46100 Burjassot, \\ Valencia, Spain \\ ${ }^{2}$ Institute of Molecular Recognition and Technological Development (IDM), Polytechnic University of Valencia and University \\ of Valencia, 46100 Burjassot, Valencia, Spain
}

(Submitted 21 June 2017 - Final revision received 19 December 2017 - Accepted 30 December 2017)

\section{Abstract}

Malnutrition is a common feature of chronic and acute diseases, often associated with a poor prognosis, including worsening of clinical outcome, owing, among other factors, to dysfunction of the most internal organs and systems affecting the absorption, metabolism and elimination of drugs and nutrients. Taurine is involved in numerous biological processes and is required in increased amounts in response to pathological conditions. The aim of this study was to describe the behaviour of taurine in well-nourished (WN) rats and to analyse the influence of protein-energy undernutrition on the pharmacokinetic (PK) parameters of taurine, using a PK model. Wistar rats were randomly distributed into two groups, WN and undernourished (UN), and taurine was administered intravenously or orally at different doses: 1, 10 and $100 \mathrm{mg}$. Population pharmacokinetic modelling of plasma levels was performed using the NONMEM 7.2 program. Several distribution and absorption models were explored in combination with dose and/or time covariate effects. Covariates such as nutritional status, serum albumin, body weight and score of undernutrition were used. A two-compartment population pharmacokinetic model with zero-order endogenous formation, passive absorption, first-order kinetics distribution and non-linear elimination with parallel Michaelis-Menten excretion and reabsorption processes best described taurine pharmacokinetics. Undernutrition acted as a covariate reducing the $V_{\max }$ of the active elimination process. Data analysis showed linear absorption and distribution, and non-linear elimination processes for taurine. Elimination of taurine was reduced in UN animals, suggesting that the reabsorption process via the secretion transporter was modified in that group.

\section{Key words: Malnutrition: Taurine: Pharmacokinetic modelling: In vivo studies}

Malnutrition is a range of deficiency states, from mild to severe, defined as a range of pathological conditions arising from coincident deficiency of protein and energy content in varying proportions, being a prevalent clinical condition in many hospitalised patients $^{(1)}$. It is estimated that $50 \%$ of adult patients admitted to hospitals have malnutrition, making it one of the most prevalent comorbidities in this population ${ }^{(2)}$. Malnutrition derives from clinical, social and cultural factors, and its association with higher morbidity and mortality rates, longer length of stay and higher hospital costs has been widely demonstrated in the literature ${ }^{(3-5)}$.

Individuals with protein-energy undernutrition have both reduced adipose and lean tissue, reductions in cardiac output with reduced hepatic blood flow and glomerular filtration rate $^{(6)}$. Considering the wide range of pathophysiological derangements in patients with undernutrition (UN), the pharmacokinetics of many of the drugs used for their treatment is likely to be affected, which may require dose modifications $^{(7-9)}$.
Taurine (2-aminoethylsulphonic acid) is a conditionally essential sulphur-amino acid. A balanced diet provides most taurine either directly or by synthesis in the liver and brain from methionine or cysteine in the presence of vitamin $\mathrm{B}_{6}$. This amino acid is eliminated in urine. As it can be formed in vivo from the metabolism of other sulphur-containing nutrients, its excretion may exceed the daily dietary intake ${ }^{(10)}$. In pathophysiological status, the endogenous production of taurine from its precursors is limited and the excretion can be incremented ${ }^{(11)}$, and thus the intake of taurine should be increased to maintain physiological levels.

Taurine is involved in numerous biological and physiological processes, including the regulation of the osmotic balance. Taurine participates, for instance, in bile acid formation and neuronal development, as well as function ${ }^{(12)}$. It exerts antioxidant and anti-inflammatory actions, as well as anti-arrhythmic/ ionotropic/chronotropic ones, besides constituting a central nervous system neuromodulator. The positive effects of taurine on thyroid dysfunction and renal oxidative damage ${ }^{(13)}$, epilepsy ${ }^{(14)}$,

Abbreviations: IV, intravenous; UN, undernourished; WN, well-nourished.

* Corresponding author: M. Merino Sanjuán, email matilde.merino@uv.es 
ischaemia $^{(15)}$ obesity $^{(16)}$, hypercholesterolaemia ${ }^{(17)}$ diabetes $^{(18)}$, hypertension $^{(19)}$, myocardial infarction ${ }^{(20)}$, hepatic inflammatory diseases $^{(21)}$ and neurotoxicity ${ }^{(22)}$ have also been reported. Hence, taurine is widely used as a nutriceutic and it is occasionally used as a drug.

Thus far, little is known about the pharmacokinetics of taurine after oral administration. Such information is essential if a regimen for administration of this agent as a nutraceutic in enteral diets or as therapeutic substance is designed - for example in treating the adverse effects of nickel in the nervous system ${ }^{(23)}$ or after paracetamol poisoning ${ }^{(24,25)}$. A review of the literature revealed only very few reports concerning the pharmacokinetics of taurine. One study performed by Rakotoambinina et al. was carried out using a constant intravenous (IV) infusion of labelled taurine at rates of either 2 or $3 \mu \mathrm{mol} / \mathrm{kg}$ per $\mathrm{h}$ with or without a priming dose in six healthy patients ${ }^{(26)}$, and in another study by Ghandforoush-Sattari et $a l^{(27)} 4 \mathrm{~g}$ of taurine was orally administered to eight healthy volunteers. Only scarce information was found on oral administration of taurine in undernutrition ${ }^{(28)}$, and no records were found about taurine pharmacokinetics in malnutrition.

The present study was undertaken to determine the preclinical pharmacokinetic model of taurine after oral and intravenous administration and to investigate the influence of nutritional status on the pharmacokinetics of this amino acid.

\section{Methods}

\section{Protocol, animals and experimental procedures}

Male Wistar rats were used in accordance with 2010/63/EU directive of 22 September 2010 regarding the protection of animals used for scientific experimentation. All the assays described in the present study adhered to the Principles of Animal Care and were approved by the Institutional Ethics Committee of University of Valencia (Spain) according to RD 1201/2005 (code A1326906234491).

A parallel study was designed in which a single dose of taurine was administered either IV or orally to each group of rats. A total of sixty-four male Wistar rats, 8-9 weeks of age, with a mean weight of 235.5 (sD 7.9) g, a mean serum albumin of 34.6 (SD 6.5) g/l and a mean serum cholesterol of 1.29 (SD 0.324) g/l were used. Animals were placed in individual polyethylene cages $(22 \times 22 \times 16 \mathrm{~cm})$ in a controlled room $\left(22-23^{\circ} \mathrm{C}, 50-60 \%\right.$ humidity) under a $12 \mathrm{~h}$ light $-12 \mathrm{~h}$ dark cycle. Subjects were randomly assigned to one of two groups: well-nourished (WN) (regular nutrition diet/WN, $n$ 32) and UN (protein-energy restricted diet/UN, $n$ 32). Both groups were allowed free access to water, but their food intake was controlled. The WN group was subjected to a standard pellet diet (2014 from Harlan laboratory) that fulfilled the normal daily requirements of a rat ( $14 \%$ proteins, dose of diet intake per d: $20 \mathrm{~g} / 251.9 \mathrm{~kJ}$ ) during an adaptation period that lasted $23-25 \mathrm{~d}$. The UN group received a diet that was altered in protein, carbohydrate and fat content (TD 99168 from Harlan laboratory; 5\% proteins, dose of diet intake per $\mathrm{d}: 10 \mathrm{~g} / 159 \mathrm{~kJ}$ ) for the same period of time.

The rats of both groups (WN and UN) were weighed on a daily basis and serum albumin and serum total cholesterol were quantified once a week using standard commercial kits (QCA Laboratory).

The main biometrical parameters used for the assessment of the nutritional status were weight and serum albumin ${ }^{(29)}$. Animals of the UN group were considered malnourished if, at the end of the adaptation period, the weight was below $80 \%$ of the weight reached in the WN animal group and serum albumin was below $23 \mathrm{~g} / \mathrm{l}$. Serum total cholesterol values were also recorded.

The pharmacokinetic study was performed at the end of the adaptation period. For this purpose, rats from both nutritional status (WN or UN) were randomly allocated into different groups based on the dose and route of taurine administration (IV or oral). This protocol resulted in a total of twelve groups, consisting of between four and six animals (Table 1).

The day before administration, rats were subjected to jugular vein cannulation with a 12 -cm-long fragment of medical-grade silicone tubing (Silastic, inner diameter: $0.6 \mathrm{~mm}$; outer diameter: $0.94 \mathrm{~mm}$; Dow Corning Co.). Anaesthesia and analgesia were induced before surgery with intraperitoneal thiopental $\mathrm{Na}$ solution $10 \%(\mathrm{w} / \mathrm{v})$ at a dose of $30 \mathrm{mg} / \mathrm{kg}$ (Dolethal ${ }^{\circledR}$; Vétoquinol) and subcutaneous butorfanol tartrate (Torbugesic ${ }^{\circledR}$; Pfizer). Under anaesthesia, a $3 \cdot 4-\mathrm{cm}$ section of the cannula was introduced into the jugular vein in the direction of the heart and the free end was subcutaneously conducted to the dorsal base of the neck, at which point the cannula emerged. The exteriorised end was then closed with a polyethylene plug. The cannula was filled with heparinised $(0 \cdot 2 \mathrm{~g} / \mathrm{l})$ saline solution. After surgery, animals were maintained in non-fasting conditions overnight with water freely available.

\section{Taurine administration and blood sample collection}

Taurine was purchased from Sigma-Aldrich ${ }^{\circledR}$ (Welwyn Garden City). A total of sixty-four animals was used in the study. Solutions were prepared by dissolving the corresponding amount of the amino acid in saline solution.

To facilitate the blood sampling of conscious rats, a $15-\mathrm{cm}-$ long silicon tube (bridge-tubing) was connected to the free end of the cannula. For oral administration, rats were subjected to gastric intubation with all the groups receiving $1 \mathrm{ml}$ of their assigned preparation.

Table 1. Experimental design

\begin{tabular}{clc}
\hline Groups & Route of administration & Taurine dose $(\mathrm{mg})$ \\
\hline WN animals & & \\
1 & Intravenous & 1 \\
2 & Intravenous & 10 \\
3 & Intravenous & 100 \\
4 & Oral & 1 \\
5 & Oral & 10 \\
6 & Oral & 100 \\
UN animals & & \\
7 & Intravenous & 1 \\
8 & Intravenous & 10 \\
9 & Intravenous & 100 \\
10 & Oral & 1 \\
11 & Oral & 10 \\
12 & Oral & 100 \\
\hline
\end{tabular}

WN, well-nourished; UN, undernourished. 
After IV or oral administration, blood samples were taken using heparinised syringes from the jugular vein cannula at the scheduled time. After each sampling time, the blood volume was replaced with the same volume of saline solution. After collection, each blood sample was centrifuged at $5000 \mathrm{rpm}$ for $5 \mathrm{~min}$ and the plasma was transferred to polypropylene tubes and stored at $-20^{\circ} \mathrm{C}$ until taurine quantification. To achieve the deproteinisation of samples, 5-sulfosalicylic acid was used as previously described ${ }^{(30)}$. Samples stored in these conditions remained stable for at least one month (the remaining taurine concentration at this time was above $95 \%$ ).

\section{Analytical procedures}

Plasma samples were assayed for taurine content by HPLC, with fluorimetric detection taking place after derivatisation with OPA$\operatorname{MPA}\left(\lambda_{\mathrm{ex}}=230 \mathrm{~nm}, \lambda_{\mathrm{em}}=389 \mathrm{~nm}\right)^{(30)}$. The buffer used to prepare the mobile phase consisted of $9 \mathrm{~mm}$ potassium dihydrogen phosphate and $0.5 \mathrm{ml} / 1$ triethylamine, adjusted to $\mathrm{pH} 6.9$ with $\mathrm{KOH}$. The mobile phase was prepared by mixing this buffer with a mixture of organic solvents (methanol-acetonitrile, 90:10) in 55/45 $(\mathrm{v} / \mathrm{v})$. A reversed-phase column was used (UltraBase C18 $3 \mu \mathrm{m}$ $100 \times 4.6 \mathrm{~mm}$ ). A flow rate of $0.9 \mathrm{ml} / \mathrm{min}$ was used, and $50 \mu \mathrm{l}$ of the clear supernatants was injected into the chromatograph. Calibration curves covering the whole range of taurine concentrations in the plasma samples (from 50 to $750 \mu \mathrm{M}$ ) were prepared in triplicate. The taurine peak area was measured in each sample and correlated with the amino acid concentration. Excellent plots correlating the peak areas and amino acid concentrations were obtained $(r>0.999)$. The accuracy and precision of the method were confirmed using four concentrations covering the range of those to be analysed. They were evaluated by calculating the relative error and $\mathrm{CV}$, which were always below $7 \%$. The limit of quantification was $57 \cdot 11 \mu \mathrm{m}$ for taurine samples. The results obtained were considered fully acceptable ${ }^{(31)}$.

\section{Pharmacokinetic modelling and statistical analysis}

A stepwise population pharmacokinetic approach was followed by using a non-linear mixed effects model and the first-order estimation method, implemented with NONMEM, version $\mathrm{VI}^{(32)}$, in conjunction with a G77 FORTRAN compiler and Wings for NONMEM. Different subroutines were used, namely ADVAN 3, 11 and 9. The experimental data used to build the model were total plasma concentration of taurine.

As the taurine concentration in plasma is owing to both internal regulation and external particular uptake, for the modelling, two input constants were considered: a zero order $\left(Q_{0}(\mathrm{mg} / \mathrm{h})\right)$ as representative of an internal equilibrium of synthesis and degradation, and a second input constant to characterise the uptake from oral administration, modelled considering a passive or an active process.

Pharmacokinetic modelling was performed from an empiric and mechanistic perspective. The empiric approach allowed deciding whether the pharmacokinetic parameters depend on the dose administered and the mechanistic approach was performed to select the kinetics of each ADME process. The model was developed sequentially, so that whenever modifications had to be made to the base structure of the model a backwards procedure was performed. Data were also incorporated in a sequential manner. The analysis consisted of the following steps:

(1) Step 1: TAU IV data from groups 1,2 and 3 (WN).

(2) Step 2: TAU IV data from groups 7,8 and 9 (UN).

(3) Step 3: TAU IV data from groups 1, 2, 3, 7, 8 and 9 (WN and UN).

(4) Step 4: TAU IV data from groups 1, 2, 3, 7, 8, 9 and oral data from groups 4, 5, 6 and 10, 11 and 12 (WN and UN).

In steps 1 and 2 , the selection of the compartmental model (one compartment or two compartments) was made and nonlinearity in the distribution or elimination processes was assayed. Step 3 was implemented to optimise the model characterising the non-linear processes detected in previous steps and also evaluating the influence of the nutritional status on the pharmacokinetic parameters (distribution and excretion). In the last stage of model development (step 4), the values for disposition parameters were fixed at those obtained in step 3, as the addition of oral data did not affect them. Then, the oral absorption process was studied and the influence of the nutritional status on taurine absorption and bioavailability was tested.

Inter-individual variability (IIV) in pharmacokinetic parameters and residual error in plasma concentration was modelled with exponential equations (Equations (1) and (2), respectively):

$$
\begin{gathered}
\theta_{i}=\bar{\theta} \cdot e^{\eta_{i}} \quad \eta_{i} \sim N\left(0, \omega^{2}\right) \\
y_{i j}=f\left(\theta_{i}, D_{i}, x_{i j}\right) \cdot e^{\epsilon_{j}} ; \epsilon_{j} \sim N\left(0, \sigma_{1}^{2}\right)
\end{gathered}
$$

In Equation (1), $\theta_{i}$ is the pharmacokinetic parameter $\theta$ of the individual, $\bar{\theta}$ the mean population value of this pharmacokinetic parameter and $\eta_{i}$ the IIV of a population $(N)$ with a mean randomly distributed around zero and an estimated variance of $\omega^{2}$. The need to include inter-IIV terms was evaluated for all parameters.

For the residual error calculation, Equation (2), $f\left(\theta_{i}, D_{i}, x_{i j}\right)$ represents the individual predicted concentration by means of a particular pharmacokinetic model $f$, with individual pharmacokinetic parameters, $\theta_{i}$, dose, $D_{i}$, and time, $j$, at which concentrations of the amino acid were evaluated (dependent variable, $x_{i j}$ ) and were modelled in terms of epsilon $(\varepsilon)$ variables. Each $\varepsilon$ variable was assumed to have a mean of zero and an estimated variance $\sigma_{1}^{2}$. A slope-intercept error model was used where the residual variability $(\sigma)$ of the exponential term was interpreted as a $\mathrm{CV}$ and the added component as standard deviation.

In the selection of the best model, the minimum value of objective function (MOFV) provided by NONMEM was adopted. For hierarchical models, the difference between objective function values is distributed as $\chi^{2}$, which allows the best model to be selected. A $P$ level of 0.01 was chosen for accepting a more complex model over a reduced one. For hierarchical models differing by one or two parameters, the corresponding differences in the objective function values are 6.63 and 9.21 , respectively. Precision in the estimation of the parameter values, quantified as the relative standard error (\%) and residual error, were also evaluated in order to select the final model. The graphical goodness of fit analysis was evaluated through the use of SPlus for Windows, version 7.0 (Insightful) and R (version R-3.1.2). 
Model evaluation was performed by means of a visual predictive check and the bootstrap resampling technique. For Visual predictive check, simulations of taurine concentration-time profiles for 200 simulated populations were performed using the final model, and each model parameter was estimated including IIV. For each group, the concentration-time profiles corresponding to the 5th, 50th and 95th quartiles were represented together with the corresponding observations ${ }^{(33)}$. Similarly, the bootstrap resampling technique was used as an internal method to validate the final model. From the original data set, 1000 replicates were generated by sampling randomly with replacement, and the final population pharmacokinetic model was fitted repeatedly to each replicate using the bootstrap option of Wings for NONMEM V package (Holford, version 222, May 2001). The mean and median parameter estimates and their $95 \%$ CI were obtained from the bootstrap replicates and compared with the population pharmacokinetic parameters obtained from the original data set

\section{Results}

Table 2 shows mean values, standard deviation and 95\% CI of serum albumin, total cholesterol and body weight at the beginning and the end of adaptation period for $\mathrm{WN}$ and UN animals. The nutritional assessment according to serum albumin and body weight is also shown. There were no differences between body weight, serum albumin or total serum cholesterol before the adaptation period. However, at the end of the adaptation period statistical differences were found in body weight and serum albumin between $\mathrm{WN}$ and UN animals $(P<0.0001)$. As previously reported, the weight evolution of UN animals was best described by linear weight gain and a decline module characterised by exponential weight loss, where the weight loss rate constant is an exponential function of time $^{(34)}$. Total serum cholesterol showed a non-statistical decrease. The nutritional assessment revealed that $81 \%(n 26)$ of the animals in the UN group developed moderate to severe undernutrition.

No statistical differences between basal taurine plasma concentration obtained in WN and UN animals were found (WN $(50.97$ (SD 14.54) mg/l) and UN (48.86 (sD 22.11) mg/l) $(P>0 \cdot 05)$ ).

Taurine plasma concentration-time profiles obtained after IV and oral administration are shown in Fig. 1. The experimental data used in the model development were taurine plasma concentration $v$. time.

A Michaelis-Menten process was fit to identify the kinetics of distribution and elimination processes (disposition phase, steps 1 and 2). The empirical model revealed non-linear excretion phenomena, but failed to provide any relevant information. Taking into account that taurine is mainly excreted in urine with high variations, a saturable renal tubular secretion and reabsorption process was tested ${ }^{(35)}$. The model with active renal excretion and reabsorption was chosen in this step. When a mechanistic approach was considered, using data from WN and UN animals (Step 3), the influence of nutritional status on Vms, Kms, Vmr and Kmr parameters was evaluated. This step showed that the active secretion process of taurine was decreased in undernutrition status; Vms was $10 \%$ lower in UN animals.

In the final step (Step 4), disposition parameters were fixed to those obtained in the previous steps and oral administration data of UN and WN animals were added. The absorption

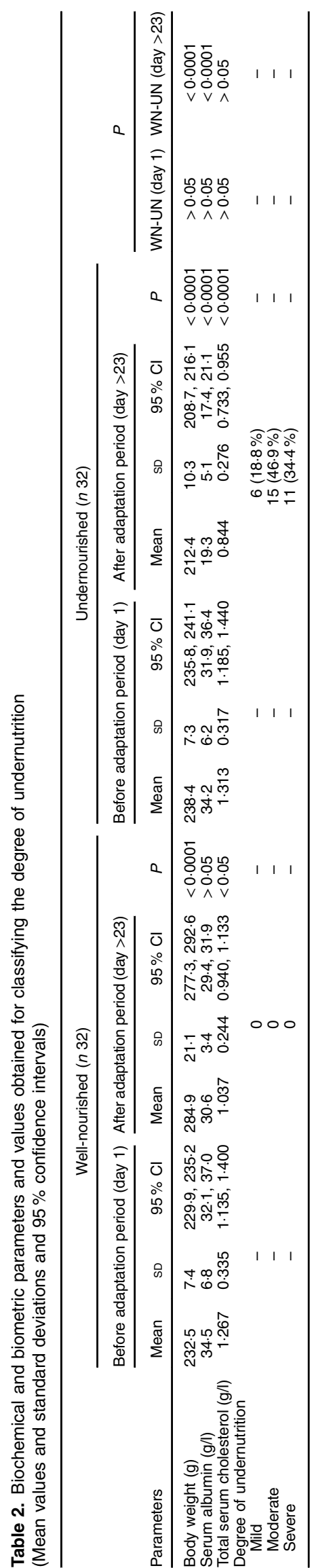



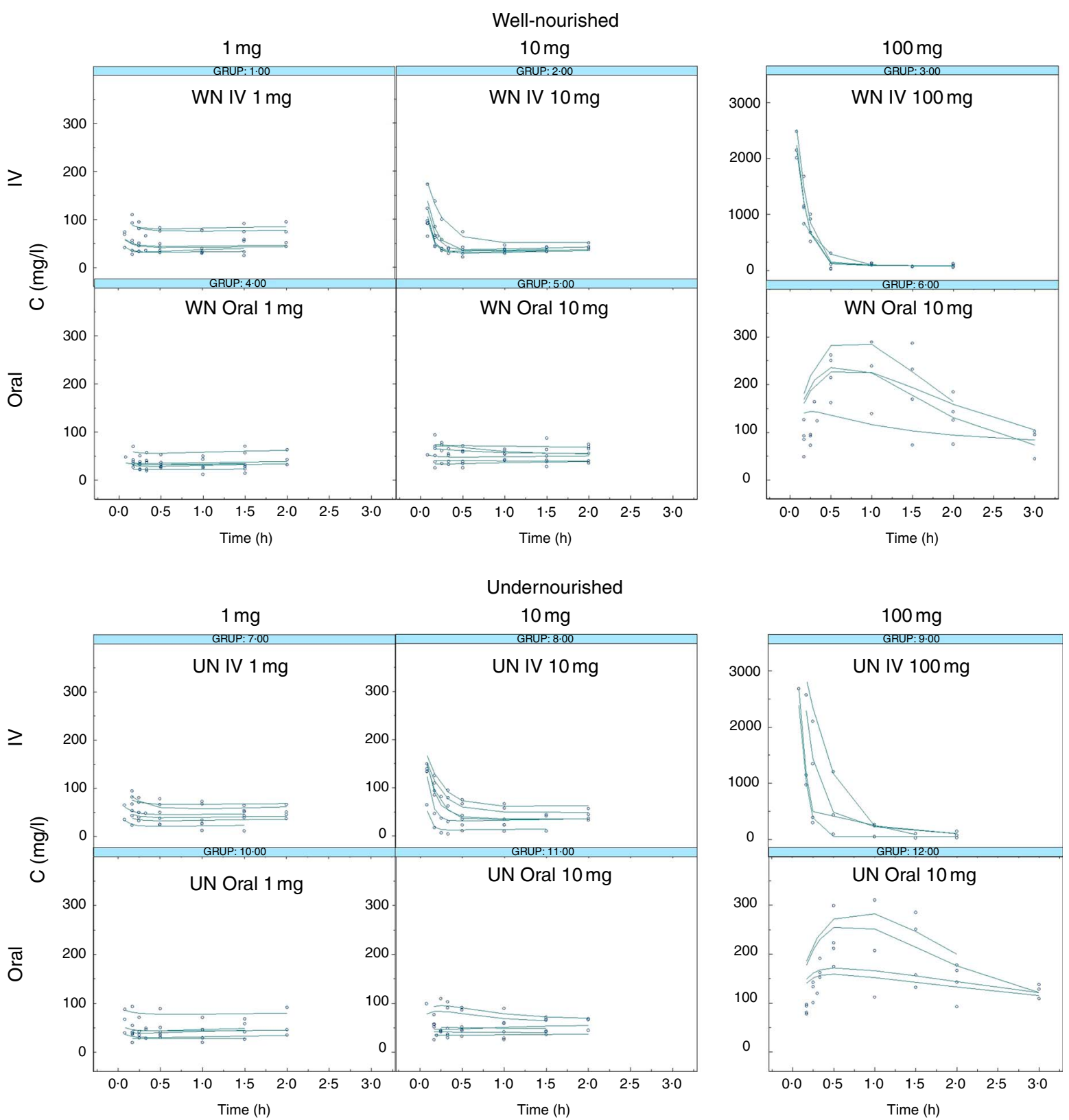

Fig. 1. Taurine levels: observed $v$. predicted. Observed taurine concentrations (mg/l, dots) and individual predicted taurine concentrations (IPREDV, mg/l, lines) $v$. time (h) according to the final model (model 150) obtained after the administration of 1,10 or $100 \mathrm{mg}$ of taurine. WN, well-nourished animals; UN, undernourished animals; IV, intravenous.

process is best described by passive diffusion kinetics. Oral bioavailability was also incorporated in the model as a function of the oral dose administered and nutritional status. In all cases, results were very similar to 1 (100\% bioavailability).

Fig. 2 illustrates the schematic structure of the model and the corresponding equation (Equation (3)) that best defined the pharmacokinetic profile of taurine:

$$
\begin{aligned}
\frac{\mathrm{d} C(1)}{\mathrm{dt}}= & Q_{0}+k_{\mathrm{a}} C(3)-k_{12} C(1)+k_{21} C(2)-\frac{\mathrm{Vms} \cdot\left(F_{\mathrm{UN}}\right)^{N U T} C(1)}{\mathrm{Kms}+C(1)} \\
& +\frac{\mathrm{Vmr}(4)}{\mathrm{Kmr}+C(4)}
\end{aligned}
$$

where $k_{\mathrm{a}}$ is the oral absorption rate, $Q_{0}$ the basal level of taurine, $K_{12}$ and $K_{21}$ the inter-compartmental distribution constants, Vmr and Kmr the reabsorption Michaelis-Menten constants, Vms and Kms the elimination MichaelisMenten constants, $F_{\mathrm{UN}}$ the reduction of Vms calculated in UN animals compared with WN animals. NUT $=0$ in WN or 1 in UN.

Table 3 shows the estimated values for the pharmacokinetic parameters of the final model (covariance step aborted). Individual taurine plasma concentration values predicted with the selected model are also shown in Fig. 1 as a line. Individual and population predicted $v$. observed taurine plasma concentrations are plotted in Fig. 3. Weighted residuals (WRES) $v$. time and absolute individual weighted residuals IIWRESI $v$. individual 


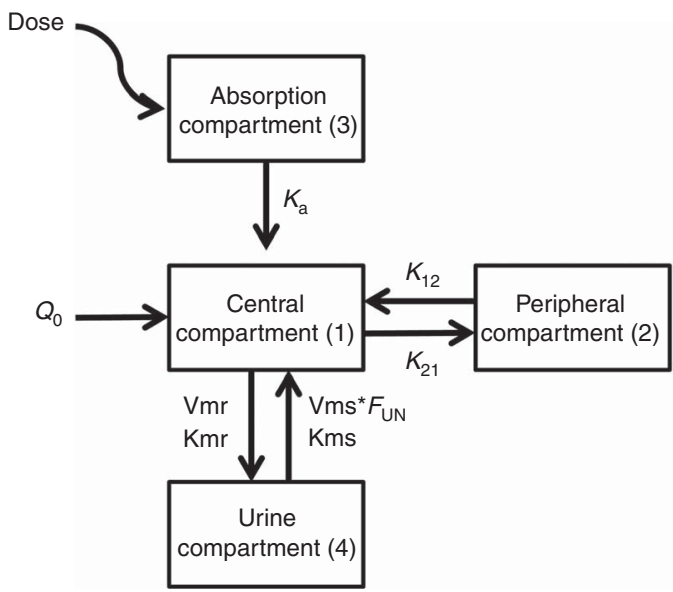

Fig. 2. Final model diagram of the final model describing the pharmacokinetic profile of taurine after oral administration. D, dose; $k_{a}$, oral absorption rate; $Q_{0}$, basal level of taurine; $K_{12}$ and $K 21$, inter-compartmental distribution constants; $\mathrm{Vmr}$ and $\mathrm{Kmr}$, reabsorption Michaelis-Menten constants; Vms and $\mathrm{Kms}$, elimination Michaelis-Menten constants; $F_{\mathrm{UN}}$, reduction of $\mathrm{Vms}$ calculated in $\mathrm{UN}$ animals compared with WN animals. NUT $=0$ in WN or 1 in UN.

Table 3. Pharmacokinetic taurine parameters for the population of the well-nourished and undernourished animals studied obtained from the original data set (left) and mean results from bootstrap analysis (1000 replicates, 811 minimisation successful, right)

\begin{tabular}{|c|c|c|c|c|c|}
\hline \multirow{2}{*}{$\begin{array}{l}\text { Pharmacokinetic } \\
\text { models }\end{array}$} & \multicolumn{2}{|c|}{ Original data set } & \multicolumn{3}{|c|}{ bootstrap replicates } \\
\hline & Estimate & $\mathrm{CV} \%$ & Mean & CV\% & $95 \% \mathrm{Cl}$ \\
\hline \multicolumn{6}{|l|}{ Structural model } \\
\hline$Q_{0}$ & $13 \cdot 7$ & $36 \cdot 0$ & 14.4 & $29 \cdot 0$ & $9 \cdot 21,23 \cdot 8$ \\
\hline$V_{\mathrm{c}}(\mathrm{I})$ & 0.0416 & 17.5 & 0.0462 & 14.9 & $0.036,0.061$ \\
\hline$K_{12}(/ \mathrm{h})$ & $2 \cdot 61$ & $44 \cdot 8$ & $2 \cdot 32$ & $27 \cdot 2$ & $1 \cdot 15,3.47$ \\
\hline$K_{21}(/ \mathrm{h})$ & 0.73 & $48 \cdot 1$ & 0.893 & $45 \cdot 5$ & $0.314,1 \cdot 74$ \\
\hline Vms (mg/l per h) & $192 \cdot 0$ & $56 \cdot 3$ & 331 & 89.5 & $78 \cdot 1,1041$ \\
\hline $\mathrm{Kms}(\mathrm{mg} / \mathrm{l})$ & 399 & 110 & 780 & 121 & $75 \cdot 8,3391$ \\
\hline Vmr (mg/l per h) & $16 \cdot 9$ & 457 & 89.1 & 228 & $0 \cdot 268,786$ \\
\hline $\mathrm{Kmr}(\mathrm{mg} / \mathrm{l})$ & $96 \cdot 1$ & 190 & 266 & 509 & $1.91,1808$ \\
\hline $\mathrm{FVms} \mathrm{DN}_{\mathrm{DN}}$ & 0.906 & $15 \cdot 1$ & 0.950 & 6.51 & $0.823,1.06$ \\
\hline$K_{\mathrm{a}}(/ \mathrm{h})$ & 1.19 & $18 \cdot 2$ & 0.969 & 23.4 & $0.575,1.45$ \\
\hline \multicolumn{6}{|c|}{ Between-subject variability (BSV) } \\
\hline $\mathrm{BSV}_{Q_{0}}(\%)$ & $25 \cdot 7$ & 104 & $20 \cdot 5$ & $51 \cdot 1$ & $0 \cdot 257,35 \cdot 2$ \\
\hline $\mathrm{BSV}_{V_{\mathrm{c}}}(\%)$ & $50 \cdot 6$ & $52 \cdot 7$ & $66 \cdot 4$ & $28 \cdot 7$ & $31.9,98.5$ \\
\hline $\mathrm{BSV}_{k 12}(\%)$ & $120 \cdot 4$ & $38 \cdot 7$ & 101 & $37 \cdot 1$ & $55 \cdot 3,156$ \\
\hline $\mathrm{BSV}_{k 21}(\%)$ & 93.4 & 293 & 109 & 118 & $0.934,326$ \\
\hline $\mathrm{BSV}_{V \mathrm{~ms}}(\%)$ & $17 \cdot 1$ & 285 & 11.7 & $86 \cdot 1$ & $0.171,30 \cdot 7$ \\
\hline $\mathrm{BSV}_{k \mathrm{a}}(\%)$ & $69 \cdot 6$ & $42 \cdot 3$ & $29 \cdot 7$ & 108 & $0 \cdot 600,82 \cdot 1$ \\
\hline \multicolumn{6}{|l|}{ Residual variability } \\
\hline$\sigma(\%)$ & $22 \cdot 4$ & $10 \cdot 0$ & 21.5 & 6.50 & $18 \cdot 8,24 \cdot 2$ \\
\hline
\end{tabular}

predictions are also shown in Fig. 3, whereas Fig. 4 shows the results of the visual predictive check.

\section{Discussion}

Malnutrition is associated with variations in drug absorption and drug disposition owing to changes in protein binging, hepatic metabolism and renal elimination ${ }^{(9,36,37)}$. As a consequence, drug toxicity increases and the response to treatment is altered. In such a situation, conditionally essential nutrients, such as taurine, that are mainly provided by dietary intake may have altered body levels. However, only a few studies have, thus far,
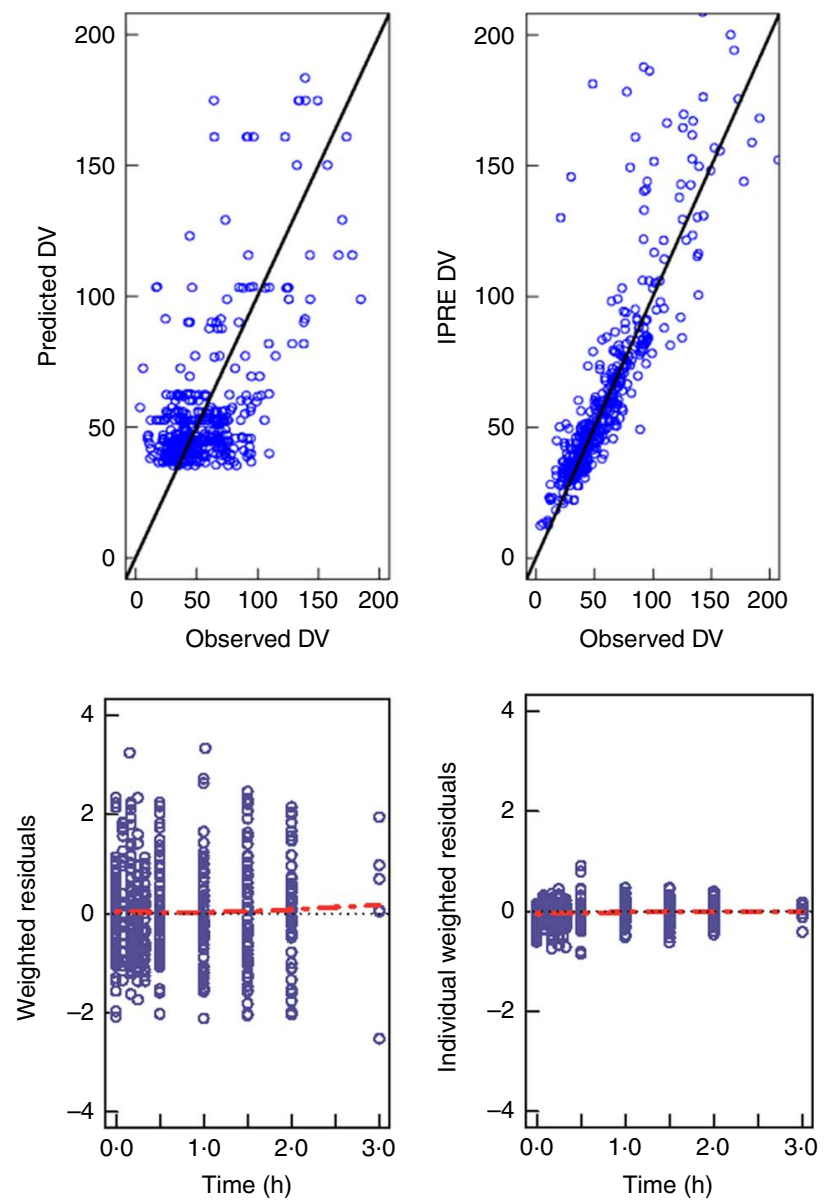

Fig. 3. Goodness of fit plots. The upper panels show observed concentrations $v$. predicted concentrations for the typical individual on the left (predicted dependent variable; DV) $v$. predictions for each individual on the right (IPRED), together with the identity line (-). The lower panels show the weighted residuals for the typical individual (WRES) $v$. time and the individual weighted residuals IWRES $v$. predictions for each individual with smooth spline (-- $)$. ........, Reference.

evaluated the influence of malnutrition on the pharmacokinetics of nutrients, such as taurine, in malnourished patients ${ }^{(38)}$.

The present study was undertaken to determine the preclinical pharmacokinetic model of taurine after oral, as well as IV, administration and to investigate the influence of nutritional status on the pharmacokinetics of this amino acid. To detect possible non-linearity, three doses of the amino acid were administered. Such information is essential when a regimen for administration of this agent to patients is designed.

The empirical models in steps 1 and 2 were fit in order to determine the compartmental model suitable for taurine and to detect possible non-linearity in the disposition processes. Both steps revealed that the model that best fitted the data was a bicompartmental model with non-linear phenomena in the elimination process, which agrees with the literature, where it has been described that taurine transport undergoes an adaptive response to changes in taurine availability and body requirements ${ }^{(39)}$. Contrary to most amino acids, taurine is not metabolised and does not bind to protein but remains free in the intracellular water. Most amino acids are reabsorbed at rates of $98-99 \%$. Reabsorption of taurine, however, may range from 

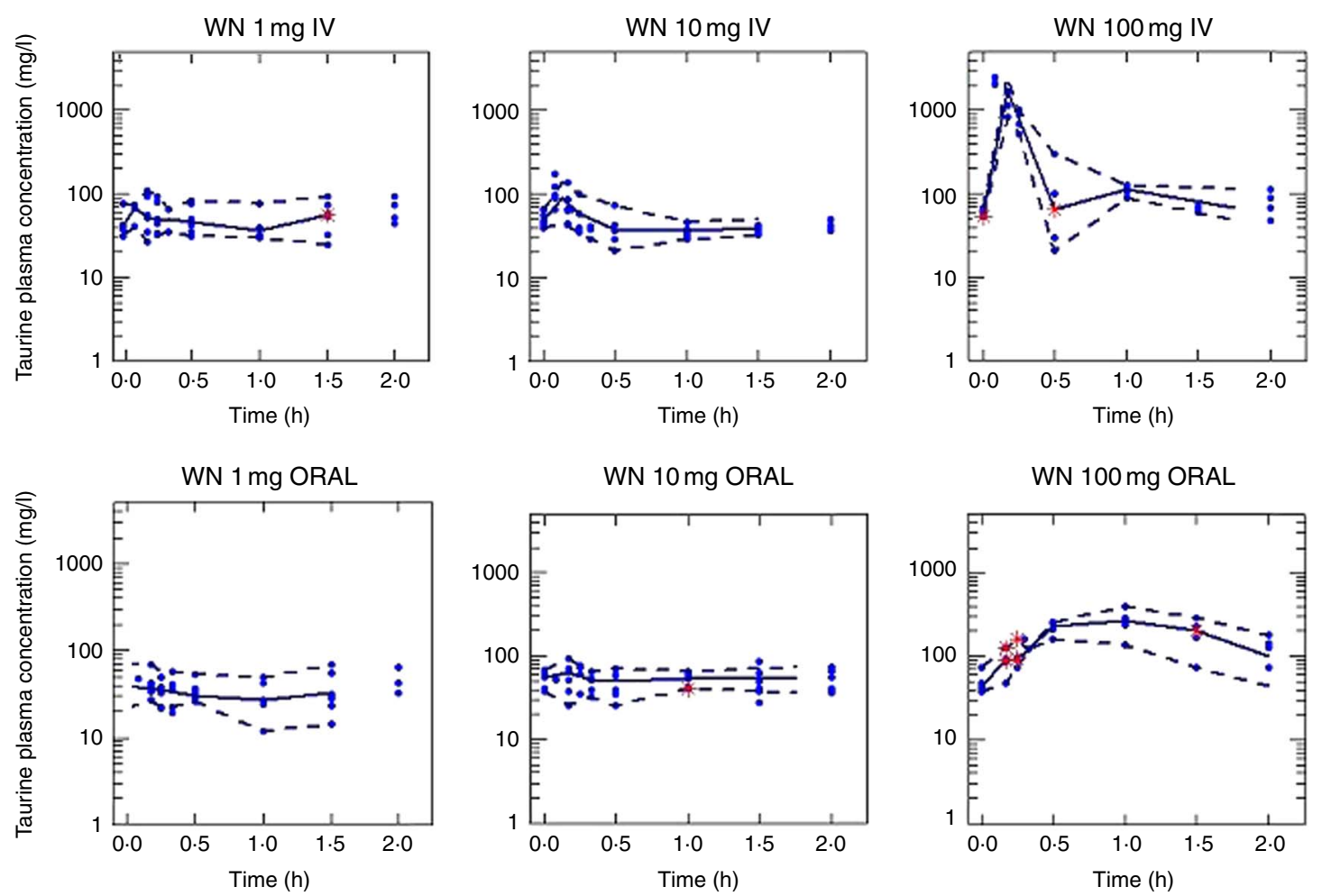

WN $10 \mathrm{mg}$ ORAL

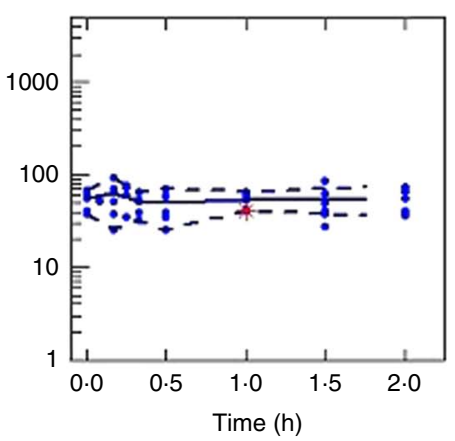

WN $100 \mathrm{mg}$ ORAL
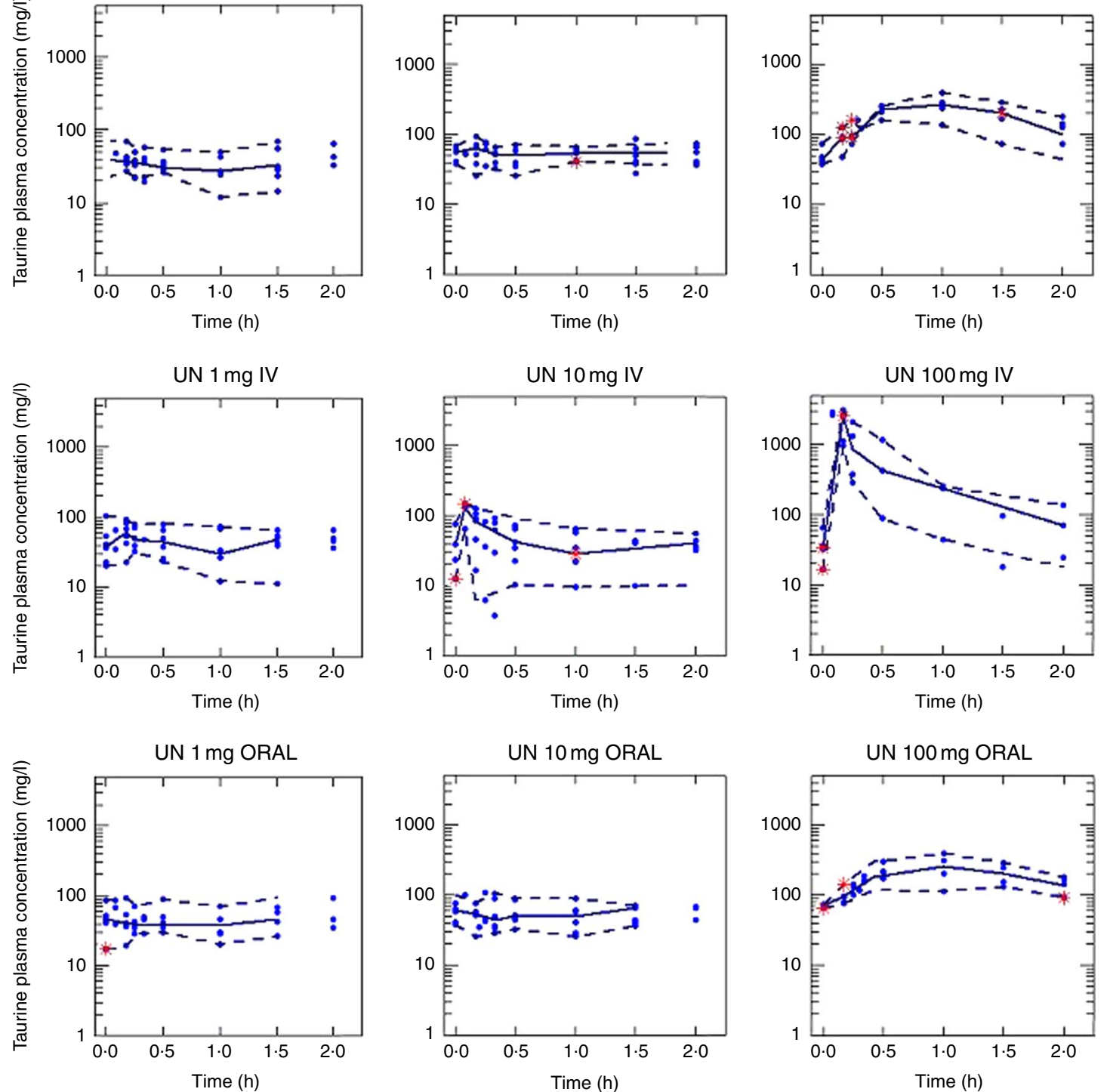

Fig. 4. Taurine plasma concentrations. Results of the model exploration exercise (visual predictive check), in which total plasma concentration-time profiles of taurine were simulated 200 times. Experimental plasma concentration of taurine $(\bullet)$. Lines represent 5 th, 50 th and 95 th percentiles. WN, well-nourished animals; UN, undernourished animals; IV, intravenous.

$0 \cdot 5$ to $99 \cdot 5 \%$, suggesting an adaptive regulation of transport in the kidney ${ }^{(6)}$. In step 3, the effect of covariates, final body weight, final serum albumin, final serum cholesterol and degree of undernutrition in those parameters, was checked to detect the influence of nutritional status on the pharmacokinetic parameters. Linear, exponential and potential equations were explored. As these analyses failed, nutritional status was introduced in the fitting as a factor. The best fit was obtained with 
the bicompartmental model with non-linear elimination, depending on both the administered dose and nutritional status.

Given the small molecular size of taurine, a passive diffusion elimination process was first evaluated. However, during the mathematical modelling of data, the successful models showed that passive renal filtration was very low $\left(<0.52 \times 10^{-9} \mathrm{l} / \mathrm{h}\right.$ per $\left.\mathrm{g}\right)$, indicating that active elimination processes (saturable renal tubular secretion and reabsorption) are significantly more relevant than passive diffusion $(P<0 \cdot 01)$. Thus, renal filtration was not incorporated in the final model. The model selected in step 3 incorporates saturable renal tubular secretion and reabsorption, thus being in agreement with previous findings. As an endogenous compound, its renal clearance is modulated according to body requirements determined by uptake and pathophysiological changes. It has been described that its elimination is because of a process sensitive to the presence of $\mathrm{Na}$ and $\mathrm{Cl}$ ions and that it is partially reabsorbed by the TauT transporter located in the proximal tubules ${ }^{(39)}$.

In this scenario, after administration of a 10-mg dose, active tubular reabsorption would be saturated, which would encourage elimination, and clearance would be higher than that observed with the 1-mg dose. Similarly, with the 100-mg dose, active tubular secretion and tubular reabsorption processes could both be saturated, so that clearance would be lower than in the case of the 1-mg dose, both in WN and UN. The influence of nutritional status on $\mathrm{Vms}$, Kms, Vmr and Kmr parameters was also evaluated in this step. The saturation of the secretion process will first occur in UN animals, which showed a $10 \%$ lower Vms than WN animals; therefore, elimination in UN animals would be lower than in WN animals. This change in clearance, which was statistically significant $(P<0 \cdot 01)$, may not have clinical relevance in higher dietary supplementation but may be of relevance in patients with normal or low taurine intake (1-10 mg each meal (or $3-30 \mathrm{mg} / \mathrm{d}$ ) in rats equivalent to $6-60 \mathrm{mg} / \mathrm{meal}$ (or $18-120 \mathrm{mg} / \mathrm{d}$ ) in humans) ${ }^{(40)}$.

In the final step, step 4, oral data from WN and UN animals were added. Results showed that the oral absorption of taurine can be better described by a passive diffusion model that is not altered by the nutritional status. The model selected to describe taurine absorption differs from other publications. Intestinal transporters for taurine (TauT) that have low capacity and high specificity have been described ${ }^{(39,41,42)}$. The differences found may be owing to the range of doses administered. The selection of the doses administered in our study was made according to the taurine doses granted in enteral diets designed for human patients (equivalent to 1 and $10 \mathrm{mg}$ in rats), whereas the higher dose $(100 \mathrm{mg}$ ) was chosen owing to its negligible toxicity. Doses of $1 \mathrm{mg}$ and $10 \mathrm{mg}$ of taurine orally administered did not significantly increase the plasma levels of the amino acid from the basal levels, whereas a dose of $100 \mathrm{mg}$ of taurine could (Fig. 1). Data obtained from the $100-\mathrm{mg}$ administration might not have been sufficient to characterise the non-linearity in the absorption process. Moreover, it cannot be ruled out that if the transporter has low capacity it can be saturated at all doses administered. Finally, it has been described that this transporter activity seems to be higher in young subjects than in the elderly; although differences in absorption were not observed, nutritional status is likely to have a higher impact in young people ${ }^{(43)}$.
The CI of some of the model parameters, which cannot be strictly considered quantitatively, is very high. Taking into account the complexity of the model, the number of available plasma determinations and that taurine is an endogenous compound, the model can be considered a good approach to describe the pharmacokinetics of taurine. Its main usefulness resides in the fact that it reveals that undernutrition impairs the ADME process of taurine. Further studies need to be conducted in order to identify more accurately the precise mechanism responsible (i.e. renal reabsorption impairment, transporters reduction and so on) in order to extrapolate these results to other species, including humans.

Two conclusions can be drawn from the results obtained in this work: the taurine absorption process at the usual range of doses apparently is passive and levels of taurine plasma concentrations obtained in this study highlight the need for augmented taurine supplementation in enteral commercial formulations to increase taurine plasma levels (usual concentrations in enteral diets: $8-15 \mathrm{mg} / 100 \mathrm{ml})^{(44,45)}$.

Overall, the selected model describes taurine pharmacokinetics in WN and UN rats at the usual doses used in clinical practice and gives an approximation about what happens when this amino acid is administered in UN conditions.

\section{Conclusions}

Data analysis showed linear absorption and distribution, and non-linear elimination processes for taurine, with active processes, renal secretion and reabsorption implied. Elimination of taurine was reduced in UN animals, suggesting that the secretion transporter was significantly modified in this group. The taurine absorption process at the usual range of doses behaves as a passive process that is not altered in undernutrition.

\section{Acknowledgements}

The authors would like to honour Professor NV Jiménez Torres for his involvement in pharmaceutical science during his lifetime. His main concern was approximating basic research to clinics in order to increase the quality and safety in patient care.

The research was financed by the University of Valencia.

The contributions of the authors were as follows: A. C. L. carried out the animal experiments and was responsible for the collection of data. A. N. contributed to data collection, the presentation and interpretation of the results and the preparation of the manuscript. V. M. participated in its design, contributed to the interpretation of the results and critically reviewed the paper. O. D. participated in the design of the study. M. M. S. conceived the study, participated in the design of the study, contributed to the presentation and interpretation of the results and coordination and critically reviewed the paper. All the authors have approved the final manuscript.

The authors have no conflicts of interest that might be relevant to the content of this paper.

\section{References}

1. Oshikoya KA, Sammons HM \& Choonara I (2010) A systematic review of pharmacokinetics studies in children with proteinenergy malnutrition. Eur J Clin Pharmacol 66, 1025-1035. 
2. da Silva Fink J, Daniel de Mello P \& Daniel de Mello E (2015) Subjective global assessment of nutritional status - a systematic review of the literature. Clin Nutr 34, 785-792.

3. Correia MI \& Waitzberg DL (2003) The impact of malnutrition on morbidity, mortality, length of hospital stay and costs evaluated through a multivariate model analysis. Clin Nutr 22, 235-239.

4. Barker LA, Gout BS \& Crowe TC (2011) Hospital malnutrition: prevalence, identification and impact on patients and the healthcare system. Int $J$ Environ Res Public Health 8, 514-527.

5. Lim SL, Lee CJ \& Chan YH (2014) Prognostic validity of 3-Minute Nutrition Screening (3-MinNS) in predicting length of hospital stay, readmission, cost of hospitalisation and mortality: a cohort study. Asia Pac J Clin Nutr 23, 560-566.

6. Chesney RW, Han X \& Patters AB (2010) Taurine and the renal system. J Biomed Sci 17, Suppl. 1, 1-4.

7. Lazzerini M \& Tickell D (2011) Antibiotics in severely malnourished children: systematic review of efficacy, safety and pharmacokinetics. Bull World Health Organ 89, 594-607.

8. Prado CM, Maia YL, Ormsbee M, et al. (2013) Assessment of nutritional status in cancer-the relationship between body composition and pharmacokinetics. Anticancer Agents Med Chem 13, 1197-1203.

9. Lykke M, Hother AL, Hansen CF, et al. (2013) Malnutrition induces gut atrophy and increases hepatic fat infiltration: studies in a pig model of childhood malnutrition. Am J Transl Res 5 , 543-554.

10. Sved DW, Godsey JL, Ledyard SL, et al. (2007) Absorption, tissue distribution, metabolism and elimination of taurine given orally to rats. Amino Acids 32, 459-466.

11. Chiarla C, Giovannini I \& Siegel JH (2003) Co-variation of plasma sodium, taurine and other amino acid levels in critical illness. Amino Acids 24, 89-93.

12. Li XW, Gao HY \& Liu J (2017) The role of taurine in improving neural stem cells proliferation and differentiation. Nutr Neurosci 20, 409-415.

13. Adedara IA, Ojuade TJ, Olabiyi BF, et al. (2017) Taurine ameliorates renal oxidative damage and thyroid dysfunction in rats chronically exposed to fluoride. Biol Trace Elem Res $\mathbf{1 7 5}$, 388-395.

14. Oja SS \& Saransaari P (2013) Taurine and epilepsy. Epilepsy Res 104, 187-194.

15. Sukhotnik I, Aranovich I, Ben Shahar Y, et al. (2016) Effect of taurine on intestinal recovery following intestinal ischemia-reperfusion injury in a rat. Pediatr Surg Int 32, 161-168.

16. Murakami S (2015) Role of taurine in the pathogenesis of obesity. Mol Nutr Food Res 59, 1353-1363.

17. Murakami S, Fujita M, Nakamura M, et al. (2016) Taurine ameliorates cholesterol metabolism by stimulating bile acid production in high-cholesterol-fed rats. Clin Exp Pharmacol Physiol 43, 372-378.

18. Ito T, Yoshikawa N, Ito $\mathrm{H}$, et al. (2015) Impact of taurine depletion on glucose control and insulin secretion in mice. J Pharmacol Sci 129, 59-64.

19. Rosca AE, Stoian I, Badiu C, et al. (2016) Impact of chronic administration of anabolic androgenic steroids and taurine on blood pressure in rats. Braz J Med Biol Res 49 , e5116.

20. Yang YJ, Han YY, Chen K, et al. (2015) TonEBP modulates the protective effect of taurine in ischemia-induced cytotoxicity in cardiomyocytes. Cell Death Dis 6, e2025.

21. Abdel-Moneim AM, Al-Kahtani MA, El-Kersh MA, et al. (2015) Free radical-scavenging, anti-inflammatory/anti-fibrotic and hepatoprotective actions of taurine and silymarin against CCl4 Induced rat liver damage. PLOS ONE 10, e0144509.

22. Ananchaipatana-Auitragoon $\mathrm{P}$, Ananchaipatana-Auitragoon $\mathrm{Y}$, Siripornpanich V, et al. (2015) Protective role of taurine in developing offspring affected by maternal alcohol consumption. EXCLI J 14, 660-671.

23. Xu S, He M, Zhong M, et al. (2015) The neuroprotective effects of taurine against nickel by reducing oxidative stress and maintaining mitochondrial function in cortical neurons. Neurosci Lett 590C, 52-57.

24. Das J, Ghosh J, Manna P, et al. (2010) Acetaminophen induced acute liver failure via oxidative stress and JNK activation: protective role of taurine by the suppression of cytochrome P450 2E1. Free Radic Res 44, 340-355.

25. Acharya M \& Lau-Cam CA (2013) Comparative evaluation of the effects of taurine and thiotaurine on alterations of the cellular redox status and activities of antioxidant and glutathione-related enzymes by acetaminophen in the rat. $A d v$ Exp Med Biol 776, 199-215.

26. Rakotoambinina B, Marks L, Badran AM, et al. (2004) Taurine kinetics assessed using [1,2-13C2]taurine in healthy adult humans. Am $J$ Physiol Endocrinol Metab 287, E255-E262.

27. Ghandforoush-Sattari M, Mashayekhi S, Krishna CV, et al. (2010) Pharmacokinetics of oral taurine in healthy volunteers. J Amino Acids 2010, 346237.

28. Batista TM, da Silva PM, Amaral AG, et al. (2013) Taurine supplementation restores insulin secretion and reduces ER stress markers in protein-malnourished mice. Adv Exp Med Biol 776, 129-139.

29. Omran ML \& Morley JE (2000) Assessment of protein energy malnutrition in older persons, Part II: Laboratory evaluation. Nutrition 16, 131-140.

30. Teerlink TVLPAM \& Houdijk A (1994) Plasma amino acids determined by liquid chromatography whitin 17 minutes. ClinChem 40/2, 245-249.

31. Karnes HTMC (1993) Precision, accuracy, and data acceptance criteria in biopharmaceutical analysis. Pharm Res 10, 1420-1426.

32. Beal SL \& Sheiner LB (1989) NONMEM User's Guides, NONMEM. Project Group. San Francisco, CA: University of California.

33. Karlsson M \& Holford N (2008) A tutorial on visual predictive checks. Abstr 1434. https://www.page-meeting.org/? abstract $=1434$ (accessed June 2017).

34. Merino-Sanjuan M, Catalan-Latorre A, Nacher A, et al. (2011) Animal model of undernutrition for the evaluation of drug pharmacokinetics. Nutr Hosp 26, 1296-1304.

35. Campos Moreno E, Merino Sanjuan M, Merino V, et al. (2007) Population modelling to describe pharmacokinetics of amiodarone in rats: relevance of plasma protein and tissue depot binding. Eur J Pharm Sci 30, 190-197.

36. Winter TA, O'Keefe SJ, Callanan M, et al. (2007) Effect of severe undernutrition and subsequent refeeding on gut mucosal protein fractional synthesis in human subjects. Nutrition 23, 29-35.

37. Saunders J \& Smith T (2010) Malnutrition: causes and consequences. Clin Med 10, 624-627.

38. Miralles-Arnau S, Nacher A, Jimenez A, et al. (2011) Impact of nutritional status on the oral bioavailability of leucine administered to rats as part of a standard enteral diet. Clin Nutr 30, $517-523$.

39. Han $\mathrm{X}$, Patters $\mathrm{AB}$, Jones $\mathrm{DP}$, et al. (2006) The taurine transporter: mechanisms of regulation. Acta Physiol (Oxf) 187, 61-73.

40. Sung MJ \& Chang KJ (2009) Dietary taurine and nutrients intake and anthropometric and body composition data by 
abdominal obesity in Korean male college students. Adv Exp Med Biol 643, 429-435.

41. Broer S (2008) Amino acid transport across mammalian intestinal and renal epithelia. Physiol Rev 88, 249-286.

42. Moll-Navarro MJ, Merino M, Casabo VG, et al. (1996) Interaction of taurine on baclofen intestinal absorption: a nonlinear mathematical treatment using differential equations to describe kinetic inhibition models. J Pharm Sci 8 , $1248-1254$.
43. Warskulat U, Heller-Stilb B, Oermann E, et al. (2007) Phenotype of the taurine transporter knockout mouse. Methods Enzymol 428, 439-458.

44. Nestlé Health Science (2015) Product composition. https:// www.nestlehealthscience.us/products/isosource\%C2\%AE-1-5-cal (accessed June 2017).

45. Abbott Nutrition (2010) Abbott nutritional online brochure. http://abbottnutrition.com.au/portals/0/img/all\%20pages\%20R1. pdf (accessed June 2017). 